\title{
Prevealence of HIV in Kaposi's Sarcoma (KS) Patients Gilbert Siame*
}

Address: University of Zambia

* Corresponding author ‡Presenting author

from 2005 International Meeting of The Institute of Human Virology Baltimore, USA, 29 August - 2 September 2005

Published: 8 December 2005

Retrovirology 2005, 2(Suppl I):PI56 doi:I0.I I86/I742-4690-2-SI-PI56

\section{Background}

A high incidence of HIV/AIDS has been observed in Zambia. Current overall estimates stand at 16\%.

\section{Method}

This was a prospective study done at a Central African University Teaching Hospital, skin clinic, for five months. 112 KS patients were recruited. Full case histories and clinical examination were taken and done, respectively.

\section{Results}

94.6\% of the KS patients were HIV positive whereas 5.4\% were HIV negative. The former had epidemic KS while the latter had endemic KS. The mean ages for the two types of KS were 34.9 years(standard deviation $=9.46)$ for epidemic KS and 34.5 years(standard deviation $=14.4$ ) for endemic KS. The peak incidence of the former type of KS was in the age range 30 to 39 years whereas for the latter type it was more or less uniformly distributed in all the age groups from 10 to 59 years, with a slight peak in the 20 year age range(hence the large standard deviation). The male(M) to female(F) sex distribution for epidemic KS was 1.4:1 while for endemic KS it was 2:1. Also, endemic KS was more common in the low socioeconomic class unlike the epidemic type which cut across all socioeconomic strata.

\section{Conclusion}

1. Most patients with KS have a high prevalence for HIV Infection (94.6\%)

2. The HIV pandemic has led to an increased incidence of KS in Zambia
3. Epidemic KS (HIV positive) is roughly equally distributed in both sexes while endemic KS (HIV negative) is twice more common in males than in females

4. There is no significant difference between the mean age of epidemic and endemic KS patients

5. Epidemic KS is distributed in all socioeconomic strata whereas the endemic type is common in the low socioeconomic class 